\title{
Reconfiguration: Symbolic Image and Language Art
}

\author{
John Cayley \\ Literary Arts, Brown University, Providence, RI 02912, USA; John_Cayley@brown.edu \\ Academic Editors: Burt Kimmelman and Philip Andrew Klobucar \\ Received: 17 October 2016; Accepted: 28 February 2017; Published: 11 March 2017
}

\begin{abstract}
Reconfiguration: Symbolic Image and Language Art' proposes an analytic and theoretical framework for computational aesthetic practices in terms of 'reconfiguration' and its derivatives, 'reconfigurationism' and 'reconfigurationist'. Digitization of the media of aesthetic practices has rendered these practices subject to software architectures derived from computational applications that, for the most part, have had little regard for aesthetics as such. The 'images' of contemporary aesthetic practices are often 'symbolic images' in the terms of the essay. They are co-produced by networked computation and digitized-symbolized-representations of media, all within new formations of ('Big') software architectures that are, typically, beyond the artists' generative, poetic control. Aesthetic practice is configured by software and digitalization. To bring art and aesthetics back into a generative relation with this potentially constrictive not to say totalizing situation, artists must reconfigure. This is an intervention that computation traditionally and productively allows, even in the era of Big Software. Reconfigurationism is demonstrated, specifically, in the field of language art and is also proposed as a poetics, characteristic of a wide range of contemporary aesthetic practice in all media where computation is at play. 'Reconfiguration' and 'reconfigurationism' distinguishes itself from theories of a 'New Aesthetic' and pretends a more insightful and critically generative analysis. The essay's 'symbolic image' bears a relation to Vilém Flusser's 'technical image' but has a clearer relation both to language and to computation, since Flusser's term is overweening with regard to (the end of the history of) language and overdetermined by its links to apparatus as opposed to the generalized abstractions of computation.
\end{abstract}

Keywords: poetics of computation; digital language art; electronic literature; electronic writing; new aesthetic; reconfiguration; configuration; programming and art; symbolic image; digitalization

'Conference for Research on Choreographic Interfaces' (CRCI) was the title of a small, studio-like conference at Brown University that took place in March 2016. ${ }^{1}$ It was organized by a faculty dancer and choreographer keen to better understand the impact of networked computational devices on the theory and practice — the culture—of embodied human movement, particularly aestheticized human movement. As such it was one of many, many such conferences that continue to be convened across the widest possible range of cultural-our focus is artistic - practices, all of which-since at least the 1990s - are called to respond to what we experience as a new cultural architecture within which all these cultural practices suddenly and ever more comprehensively find themselves dwelling and working and making. Despite the fact that this networked and computational architecture is more or less ubiquitous in the developed and developing worlds, organizers of such gatherings still bring together participants who tend to be outliers or avant-gardists in their practices, specialists who have been diverted from more conventional trajectories by the promises and perils of 'digital media'. Moreover, expertise in networked computation may trump certain commitments to a particular

1 The conference took place from 4 to 5 March 2016 and was organized by Sydney Skybetter http:/ /www.choreotech.com. 
practice-ostensibly choreography in our anecdote-that is supposed to be bringing participants together. The interests, engagements and actual practices of our participants may be wildly disparate.

So, in this venue, the question is asked, "What is it that our practices share? What is it that we all do?" This is a difficult question to answer, particularly difficult in the form of a word or phrase that might indicate a shared medium, or characterize an artistic school, or serve as an agreed term to which practitioners, critics, and theorists might refer. I am a writer, and I was invited to this conference because-as I saw it-I work with networked and computationally enabled transactive synthetic language. Such language is beginning to be quasi-autonomously sited in devices which, precisely because they simulate human transaction in aurality, and because such transaction is definitive of human embodiment, should, indeed, be considered as choreographic interfaces. As a participant at the CRCI, however, I remained a writer, not a choreographer or dancer. I attempted, awkwardly, to formulate an answer that would span the disparities.

"We are the artists whose media—by which I mean the plural of medium—are being reconfigured before their eyes, within their hearing, under their feet, in their very hands. Our practices share an urgent need to respond to these circumstances, because they present us with crisis, catastrophe, pharmakon, existential challenge." ${ }^{2}$ The implication was and is that digitization and digitalization have, historically, produced a reconfiguration of all media. ${ }^{3}$ Artists do, of course, experience this as novel (as, indeed, 'new media'), as stimulating, as opportunity. At the same time, they confront its effects as interruption, disturbance and, perhaps at best, reconfiguration, where these circumstances have impacts on the very substance and materials with which they work, with which they make their art. The crisis-these circumstances as pharmakon, as both poison and potential therapy-arises from the once again historical evidence that this fundamental reconfiguration is driven by forces that are overdetermined by neoliberal modernity, focused on scientistic economic values, on the reconfiguration of cultural and social welfare as subject to principles of efficient, market-driven resolution-where resolution is self-referentially defined within the enclosed logic of neoliberal economics and the contemporary regime of computation that provides its services, while building this same logic out into the 'civilized' world as Big Software architecture [3].

An example with regard to my own current practice. Technological developments in natural language processing (NLP), digital audio, segmental analysis-automatic speech recognition and speech synthesis-have given rise to what I call transactive synthetic language (TSL). This is rolled out to the general public in the service of retail networks, focused on the ideals of frictionless commerce and consumerism, while discovering or anticipating, in the process, its 'killer application' to domotics, easy control of the 'smart home' [4-6]. Nonetheless, TSL becomes perceptible to me as a fundamental reconfiguration of linguistic production and reception, of language, of my medium as a writer, and it is available to me as affordable hardware connected to low-to-no-cost network functions. Moreover, the top-level service providers have gifted us with an application programming interface (API) for the 'voice services' in question-I can make my own transactive linguistic artifacts within and across this network of actors and affordances. Any such work that I make will, necessarily, be implicated with all the other-many unforeseen-consequences of the underlying systems and networks. Amongst the most striking of these is surveillance-otherwise cast as the collection of anonymized 'data', the benefits of which are reflected back to us in the guise of Big Data once this is owned and aggregated by the service providers, thus making themselves and us—supposedly-better, where the only real certainty is that this process makes them richer and us better at making them richer [3]. Meanwhile

2 This reference to and usage of pharmakon is inspired by the critical thought of Bernard Stiegler. See, amongst many other references [1,2].

3 While digitization might be used for both senses, digitalization may also be deployed to indicate, generally, institutional and social reconfiguration and accommodation to digital culture and networked computation, whereas digitization may have a constrained sense: the encoded representation of information about the world in digital form. 
our understanding of surveillance and marketing and-to get back to my own concern-language has changed, fundamentally.

But as an artist-maker in these circumstances, with beautiful new materials to work with, what can I do? There is a kind of imperative, regardless of the consequences, just to 'get stuck in' and make something. When it comes down to answering the same, 'what can I do?' but in terms of what it is possible for me to do, we return, I believe to reconfiguration, this time to reconfiguration as an aesthetic, generative response to the reconfiguration of media that has been imposed by networked computation. It is the fact that my newly chosen medium is a technologically mediated reconfiguration of my underlying medium which, itself, imposes a constraint on my subsequent practice. What I make with the newly reconfigured language, with TSL, will be made by reconfiguring the computational networks that have brought TSL into existence, not, that is, for example, by building a new and separate computational network for TSL. Even were this to be possible for me or my fellow artists, this would still amount to a responsive gesture, essentially a highly redundant and culturally isolated response that would, in the end, amount to no more than the kind of reconfiguration that actually is within my, our range of possibilities.

At the CRCI, after some further discussion and in part-response to my remarks, a participant suggested that we might call ourselves, collectively and as characteristic of the practices we share, 'the reconfigurationists'. ${ }^{4}$ This essay is already an exploration of what this might mean. In what follows, briefly, I will discuss, most specifically, certain aspects of poetic reconfiguration in language art. But I will also discuss reconfiguration as a poetics of computation and of networked computation in general. Within 'reconfiguration' and 'reconfigure' we easily discover 'configuration', 'configure', 'figure', and even 'figuration'. The 'figure' at more or less the root of these words, I will discuss in terms of what I call 'symbolic image'. This term bears a certain relation to Vilém Flusser's 'technical image' but rather than proposing a transcendence of writing and history, constrains its application to what we perceive as the figureable content of aesthetic artifacts regardless of medium, and the 'symbolic' qualifier is taken to further restrict our attention to images that are co-produced with the symbolic processes of (networked) computation [7,8]. We are used to the proposition that 'images' as such may come to our critical attention when we experience an aesthetic linguistic artifact-a poem, a short story. Analogously, once language has been reconfigured by networked computation, a reconfigurationist writer may compose the kind of linguistic artifact by means of which we may experience 'symbolic images' in the sense that I will begin to elaborate. I will also compare the properties and methods of my proposed 'symbolic images' and reconfigurationist artifacts with those that are associated with the 'New Aesthetic', one of the few terms in current art-critical discussion that addresses a poetics of computation explicitly. ${ }^{5}$

Critical discourse is conversant with a specialist, mildly metaphoric usage for the term 'image' such that it may be applied without regard to the medium in which an image is expressed-an image in language, an image in music. Nonetheless, in the discussion of New Aesthetic, actual visual examples tend to predominate, and these visual images (often also 'visualizations') may be marked by perceptible breaks in the visual field that are the trace of computationally generated artifactuality (although usually referred to-and I consider this a misdirection-as 'virtual' or 'virtuality'). I characterize these breaks as media-constituted and have, previously, analyzed them in terms of diegesis [11]. For a particular New Aesthetic visual image (in both senses of 'image'), for example, there may be diegeses constituted by photo-naturalism and by computer-generated graphics. A quick internet image search for 'New Aesthetic' will bring up many images within which this distinction is clear and marked-computer

4 It was Patrick Corbin, a prominent dance artist and professor of Contemporary Dance at the Kaufman School of Dance, University of Southern California, who suggested this term.

5 James Bridle is credited with coining the term, for which there is a Wikipedia entry [9], leading to a number of manifestations by Bridle, including [10]. 
graphic 'sprites' are found sharing the visual field with conventional color photography but clearly arriving within this field from 'another world', another media-constituted diegesis.

The work of Clement Valla is frequently cited as an example of the New Aesthetic and I can illustrate what I mean, in a more subtle form and one more broadly applicable across media (mediums), in a brief discussion of his Postcards from Google Earth [12], using the same illustration as that in the engaging manifesto for the 'New Aesthetic' by Curt Cloninger [13] (see Figure 1).

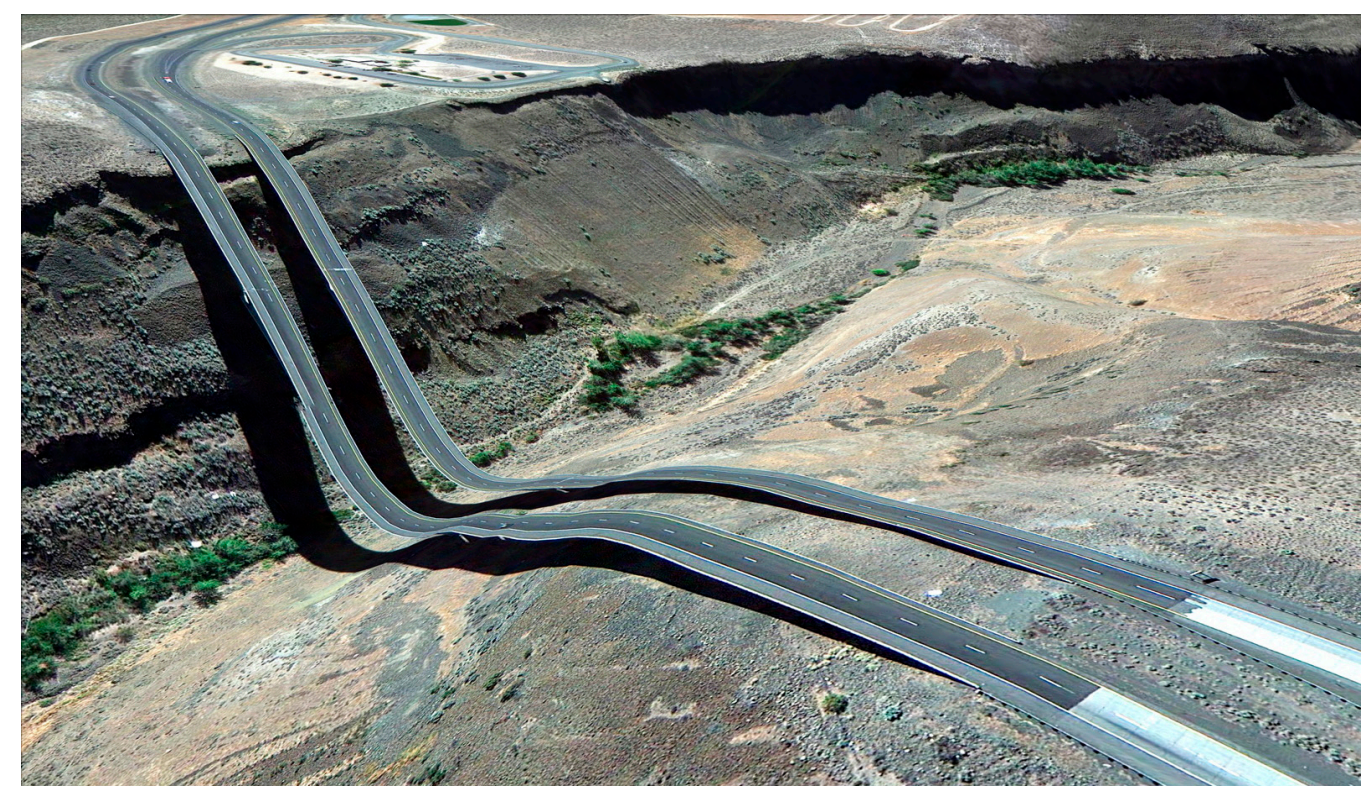

Figure 1. One of a still growing archive of images—this is tagged 'redmon'—from Postcards from Google Earth by Clement Valla, 2010. Reproduction courtesy of the artist.

Valla finds 'sites' in Google Earth where the algorithmic transformation of photographic satellite imagery into 3D images - to be projected onto our 2D computer monitors - appears to fail or, rather, produces a strikingly unusual image. He takes snapshots of these sites and turns them into 'postcards'. Presumably, altitude and contour data for the satellite images is used to produce 3D transforms and this 'fails' - as in the image illustrated — when pixels that photographically represent what is typically an architectural object, such as a bridge, are treated in the same way as pixels that represent their underlying topography, which is the source of the altitude and contour data. On the face of it this presents itself to the human viewer as error or as 'glitch', and Valla's-along with much digitally mediated art-is sometimes mistakenly associated with what is, in fact, a quite distinct 'glitch aesthetic' in computational practice. It is up to the theorists of the New Aesthetic to clarify its relationship to glitch. In setting out from a New Aesthetic analysis but denying 'error' as significantly or affectively operative in a work such as Postcards, my aim is to propose symbolic image as a more appropriate term for work of this kind and a better analytical starting point.

For the composition and appreciation of Valla's Postcards, there are still (at least) two media-constituted diegeses in my terms: that of the source satellite imagery (a kind of remote photo-naturalism), and that of the 3D-for-2D transform. The latter is the product both of digitization-the photographic data's abstract symbolic representations as encoding, and of algorithmic manipulation-processing on the basis of this abstract symbolic representation in terms of regular computational operations that may be considered typical of contemporary cultural symbolic practices. On both counts, the adjectival qualifier 'symbolic' may be considered appropriate, and therefore I say that the entire visual field of a Valla postcard itself constitutes a symbolic image. The misperceived 'glitch' is, rather, a function of the typical and regular properties and methods of symbolic imagery. The apparent mistreatment of the bridge is actually, in a sense, attributable to the failure of photo-naturalism (and its associated 
metadata) to distinguish a break (easily perceptible to humans) between the media-constituted diegesis of architecture and that of natural topography. ${ }^{6}$

So, we may say, the 'figure' of Valla's Postcard, its 'figuration', is a symbolic image, and it seems to me appropriate to further delineate such an image as, typically, also a 'configuration' since it must be composed in conjunction with other figurative, image-generative systems. While figurative systems of this kind are far from new - photography and cartography are, of course, figurative systems that have, over a long period, been configured for aesthetic making-historically, networked computation has led to the proliferation of such systems and has guaranteed their ever increasing cultural reach and momentum. Furthermore, the practice of computation has, itself, elaborated a special and appropriate sense of 'configuration' - as the Oxford English Dictionary puts it, "To choose or design a configuration for; to combine (a program or device) with other elements to perform a certain task or provide a certain capability", with the earliest quoted usage of this kind dated 1965. While the production of symbolic images may require coding and custom software, it is almost universally the case that such artist-authored programming performs what I am identifying as configuration with respect to one or more existing, usually larger, systems. Since the early years of the 21st century, such systems have, as I suggested above, come to constitute the Big Software architecture of our civilized culture in so far as it is mediated by networked computation. Configuration may, therefore, be proposed as the name for an aesthetic practice having its own distinct poetics, for the generation of symbolic images that are intended to inform and delight us with regard to our newly mediated cultural experience.

Then, finally, the justification for the 're-' of 'reconfiguration' relates to the fact that there is a determinative and, for the time being, unilaterally, inequitably, and hierarchically implemented configuration that Big Software imposes on cultural practices in circumstances that were referred to earlier. For the artist, I have suggested, their media (mediums) are now subject to substantive configuration by software architectures and for certain practitioners and theorists-if not for all of us-this is experienced as a crisis, a pharmakon, an existential challenge. A large part of aesthetic making will be, in these circumstances, stimulated by an imperative to respond, to react, and to engage in a poetics of, I propose, reconfiguration in order to generate those symbolic images that are demanded from us as part of a broader cultural poetics that is commensurate with networked computation, one that bears-one would hope-enough significance and affect to influence the trajectory of ongoing historical processes that might otherwise render us artless and careless.

When we discussed Clement Valla's work with the reconfiguration of contemporary digital photography and remote imaging in his Postcards, ${ }^{7}$ it was important for us to remark that although this work is based on the configuration of remote photo-naturalism and Google's distinct algorithmic processes that attempt to render this remote photo-naturalism as $3 \mathrm{D}$-for-2D, the entirety of the visual field of a postcard is subject to the same regular processes of first more or less conventionally understood photographic apparatuses and then algorithmic processes. The image is an integration, a synthesis of what may also be seen as two media-constituted diegeses and the misperceived 'glitches' - the apparent, human-perceptible disruptions and distortions in the postcard images-are actually to be referred back to a more striking diegetic break in the subject of the initial remote images (topography vs. architecture). In the materiality of the postcard image itself-which I call a symbolic image for the reasons of configuration given-there is no break: its pixels are

6 In terms of the type of analysis that I am outlining here, Valla's project exemplifies another media-constituted diegetic break that is interesting although less immediately important for our argument. This is the break between, as it were, the diegesis of the snapshot or postcard and that of the mapping system that is represented by Google Earth. The break is, of course, visually manifested by the framing of the postcards themselves, breaking them out of the map and its transformations. His insightful, aestheticized exposure of the contrasting ways that human participants relate to these two diegetic worlds and act with and within them is, of course, an important impetus for Valla's project.

7 For subsequent projects, Valla has contextualized his process in terms of photogrammetry, a pre-computational analytic practice of photography and clearly also productive of symbolic image in the terms set out here [14]. In the Surface Proxy catalogue cited, see especially the 'Notes' on pp. 79-94. This catalog is available in PDF form [15]. 
positioned and colored as a function of image-generative symbolic synthesis. It is important to remark this characteristic lest we be seduced by those visual images in which there are marked and obvious-'graphic'-media-constituted breaks into thinking that this is what sets them apart as, for example, New Aesthetic, leaving work in which the visual field appears 'unbroken' out of account. In my terms, this makes it more difficult to distinguish 'glitch' from symbolic image and, more importantly, it prevents us from discovering the commonalities of reconfigurative practices across other media (mediums). Within a single auditory experience of music, for example, the 'worlds' of distinct stylistic musical 'diegeses' maybe sharply distinguishable to trained ears, while for other, less cultivated, listeners a single musical world is experienced. The human ear's unfiltered, minimally directed, fundamentally continuous experience of sound as the medium of music is reasserted for those listeners without an apparatus to experience it as articulated by distinct stylistic diegeses. As we turn our attention to linguistic artifacts in order to explore certain ways in which symbolic, algorithmic process may generate symbolic images in reconfigurations of language as the medium of the work, we should first consider certain media specificities of language.

Language only ever exists as a function of readability, or, minimally, as a function of bearing some kind of significant and affective relationship to readability [16]. What we read are grammé, distinct units of inscription, at various levels of linguistic structure. These units of inscription are only perceptible, and thus readable, as distinct from whatever else they are not, and this 'whatever' could, in principle, be made of anything material, although it is usually either aural (vocalized sound to be read as speech) or visual (graphically inscribed writing). Language comes into being-into the world of human experience where we may use it as a medium to make other things-as a function of a fundamental media-constituted break with its own material supports and also as a function of continually reasserted breaks between all of its constituent elements.

It is important to set out this aspect of language's singularity for at least two reasons. In the first place, because this means that any and all higher-order (media-constituted) diegetic breaks within a linguistic artifact between conventional linguistic images and, for example, forms of language generated by algorithms or symbolic processes will always coincide with one or more of the lower-order symbolic breaks that constitute language as such. It will never be absolutely clear-from the material support of the language in question-whether a particular break occurs to mark the intervention of symbolic process or simply because the break is required in order to allow its material forms to be read as language. ${ }^{8}$ Secondly, the differences that constitute language as such are structurally the same as those that constitute the regular formal 'languages' that are used for the transcription of algorithms, programs, and so on-for the inscription of the symbolic processes that enter into the synthesis of

8 As language, these high-order breaks, as we will see, may be discovered by close reading. They may also, of course, be explicitly marked by punctuation - whether this be more or less conventional punctuation or distinctly paralinguistic. For example, within the scope of graphic inscription and typography, such a break could be marked by a change of font or a change of a single font's color. These marks, however, are at best paralinguistic; they are distinctions inscribed into the material support of the language rather than into the language as such. When it comes to language in aurality-as human or humanoid voice- the situation becomes more complex because we cannot (yet) conceive of the voice that is not marked by human individuality. This implies that whatever a voice inscribes is, minimally, within the diegetic scope of this individuality. If an apparent individual is subject to symbolic process for the production of their language, would this not break their individuality and require a change of voice (perhaps expressed as distinct intonation or accent-think also of acting, drama, and the complexities that this field of aesthetic practice would further introduce)? These are questions-somewhat beyond the present scope of this essay-that become crucial since the advent of distributed entities that speak and listen-such as Siri, Cortana, Google Now, Watson, and Alexa, all of which are literally embodied as transactive synthetic language. The language of these entities is symbolic image in the terms delineated here. It is a synthesis of conventional linguistic image and language-generative symbolic process. Not only are the broken diegeses of this language disguised by their inevitable coincidence with differences that constitute language as such, synthetic language in aurality must also be wrapped within one of the definitive indications of human embodiment, an individual voice. This renders the implicated symbolic processes compelling in so far as they acquire a compelling relationship with embodied humanity. The resultant voices are not, by the way, necessarily 'uncanny' (disturbingly human-seeming non-human). They are something more troubling than that. They are signs of the advent of a new kind of transactive being that is able to share our language-making and language-reading. 
symbolic image. ${ }^{9}$ As such, not only can the virtual linguistic output of symbolic process be seamlessly intermixed ('seamfully camouflaged' would, ironically, be more accurate here) with conventional linguistic images, but 'tokens' and 'snippets' of the regular formal languages that are used to transcribe symbolic processes may also be intermixed with natural language without necessarily exposing a break in the material form of a hybrid, intermixed linguistic inscription. This can give rise to serious confusions concerning the characteristics of certain contemporary linguistic artifacts and can make the task of close reading such artifacts complex and difficult. ${ }^{10}$

The poetic generative work of Nick Montfort demonstrates what I mean along with other characteristic of the symbolic image in language art practice. ${ }^{11}$ Montfort's work is simultaneously literary and programmatically virtuosic. It is also often minimalist and therefore relatively easy to read. In PPG256, Montfort composes the formal language of Perl into tiny programs (256 characters or less) that encode an astonishing measure of significant and affective English poetic compositional procedure [23]. Many of Montfort's tiny programs are designed to run endlessly (i.e., regularly, computationally), generating virtual language that is not only readable; it also expresses patterns of aurality-targeted sound and proto-semantic sense that may be referred to the formal rhetoric of poetry. These texts are, typically, spewed out in verses or fragments that present themselves as, simply, pieces of language. Any diegetic break between, on the one hand, the regular encoded manipulation of abstract literal tokens, and on the other, poetic language (that might be the composition of a human author) is smoothed over by the language itself and its presentation (as continuous lineated output). We can read the major break that does dwell within and constitute the work by close reading both program and output. Deconstructing the programmatic Perl we may understand exhaustively how the patterns of tokens are assembled. Close reading the output, while much of it is poetry, we agree, we will also often find lines and passages where abstract pattern generation fails to produce anything that

9 The 'language' of 'formal languages' is within quote marks here because I would prefer to reserve 'language' for natural human language. Otherwise, the use of 'language' is inflated, as Derrida pointed out long ago ([17], p. 6). Formal 'languages' are exhaustively describable in structuralist terms such as sign, grammar, difference-structure, math, logic. The post-structuralist critique, especially as emerging from Jacques Derrida's grammatology, underlies my thinking throughout, where, for example, difference must be-and must also be superseded/supplemented/erased by-différance, but only in so far as this is readable as generative of those kinds of meaning that are vital for human animals.

10 There is, of course, a reference to long-standing and ongoing discussions of the interrelationship of code and text in, especially, the field of electronic writing, digital literature, etc. The deliberate, aesthetically or conceptually motivated, synthesis of literal code and text is and will continue to be practiced-notable practitioners: Mez Breeze, Talan Memmott, Alan Sondheim, the last of whom is credited with a term for this practice, namely 'codework' [18]. The author has made his own contribution to the debate [19]. As an example of a highly interesting and conceptually superlative form of the practice, I would like to cite William P. Hicks' as yet unpublished Esopo project [20]. Hicks' approach is to specify formal languages-ideally languages that are Turing complete; that is, languages that are capable, in principle, of computing anything computable- on the basis of the selected features of existing natural linguistic textual forms. For such an approach, aspects of punctuation-particularly actual punctuation 'marks' and also the significant arrangement of 'white space' such as lineation, spacing, and indentation—are crucial, but selected textual 'tokens' — typically to be defined as 'reserved words'-will also figure in the specification. The goal of a particular constituent language within this project might be, for example, to implement a specification that will allow any text that is formatted according to genre conventions or, indeed, any text at all, to be successfully compiled into a machine language executable. In other words, certain texts are rendered interpretable-by the specification - as the 'high level' source code of a program that will compile and run on some arbitrary hardware platform. Hicks has, indeed, made a specification that will allow the compilation of any text, but he has also specified an inherently more interesting language, 'Emily,' that is able to interpret and compile any of the poems of Emily Dickinson, and thus also, by definition, any original poems that follow the textual and poetic conventions of Dickinson's work. Success in the compilation of a text is a necessary condition for it to be considered as conforming to an Esopo language specification, but Hicks' proposal is that there are expressive and aesthetic potentialities in refined instances of the practice. A language that allows any text to be compiled must allow that the vast majority of texts will compile as programs that do little more than nothing. Most of them will be instances of "Hello World," one imagines. Hicks, however, writes that 'Emily' compiles Dickinson poems as "non-trivial algorithms." Hicks conceives of a constrained writing practice-self-reflexive with regard to its own structures, which are implemented as an Esopo-that would bring into existence texts that do something significant and affective in the worlds of both human reading and machinic computation. To quote Hicks himself in [20], "Some [Esopo languages] are designed to compile almost any text they receive as input, while others demand strict adherence to certain formal rules. Other languages are designed not so much to encourage production of new algorithmically-engaged text as to draw attention to the structure of existing work."

11 A good way into Montfort's work is [21]. The author has also written a review essay of this book that deals with a number of related issues in the theory and practice of digital language art, computational poetics, and the poetics of computation [22]. 
enough of us would agree to call 'poetry'. These instances of virtual language are then, just that. They fail to be readable as or in order to become actual language and thus they serve to make visible (beyond any material form of the intermixed linguistic output) the break between generative proto-linguistic pattern and language as such.

For, when our attention is turned to linguistic artifacts-with regard to which we have proposed that any perceptible breaks in the material form of the artifact are masked or doubled by breaks that are constitutive of the artifact's very medium - the mark of a higher order break (or its synthesis) will be discovered by the perception of dissonances between the actual syntax of the artifact and natural linguistic models of syntax (grammar) that have been cultivated by the human reader. It is not only difference/différance that is constitutive of natural language. Not in any case for the common human reader. Grammar, extending to word choice, to diction, is also required. This is one of a number of reasons that symbolic image in language is resisted by readers despite the relative media-specific 'invisibility' of its breaks. If language that 'looks like language' on the surface proves to be recalcitrant, it may be considered to be not only 'broken' (and thus, perhaps, redeemably readable as the synthesis of conventional linguistic image and symbolic process); rather, it may simply be considered and judged as not (actual) language at all.

In certain branches of my own practice that I would be comfortable analyzing in terms of symbolic image and reconfiguration, I work together with symbolic process in order to smooth over the kind of breaks that might otherwise incline my readers to judge my linguistic artifacts as 'not language' in this way. [n-gram] Loose Links are quasi-algorithmic micro-collages ([24], pp. 34-57, 138-39). They are constructed around the concept of the 'longest common phrase', as developed in the context of The Readers Project [25]. A longest common phrase is, for any attributed text, a sequence of its words that can be found elsewhere in a multi-author corpus and not attributable to the original author, thus proving, minimally, that it is still an attested, discoverable part of the commons of language. For the Loose Links these provide a model for the links in its quasi-algorithmic processes of serial micro-collage. This is the opening part of 'I had a visit today ... ':

I had a visit today, for monitoring, from almost the only group that ever comes to me, rather than me going to them. I needed to make it about them and their needs, not about me and my needs. I needed a new atmosphere, a new environment, and I found it and I'm extremely excited and happy: people with bipolar disorder will have a mixture of negative and positive feeling all at the same time, and in time, and in your own time, etc. ([24], p. 35)

The typical longest common phrase is-currently, in natural language corpora of English-between three and five words in length. 'I had a visit today ... ' starts by internet searching for results containing the first four words of its title and proceeds with searching for another similar length phrase that had been contained in the first author-selected result, and so on, with another and another phrase in the selected results of subsequent searches, linked by the search phrases to compose the final text. The processes deployed are quasi-algorithmic in that they require the regular computational services of internet search and also because the micro-compositional procedure could be set out as looping pseudo code, with a human author intervening for the selection of particular results and also of subsequent linking phrases. In this work the length of the phrase all but guarantees-sometimes with a little tweaking — syntactic continuity across the linked language. In the passage quoted there are five different results and the linking phrases are very difficult to determine. The piece is an assemblage of found natural linguistic images. As a whole, it is symbolic image because symbolic processes engaged with syntactic continuity are used both for networked computational search and also in order to link the found linguistic images together. Ironically, this particular process also 'heals' what might otherwise have been instances of grammatical dissonance indicating the main constituent diegeses within the symbolic image. In terms of reconfiguration, internet search configures the potential discovery of the natural linguistic images and, as a language artist, I have reconfigured this Big Software cultural architecture to produce my reconfigurationist symbolic image in language. 
Francesca Capone's Primary Source provides us, in conclusion, with an example of reconfigurationist symbolic image, the production of which is more clearly, and in the contemporary moment more typically, involved with prosthetic technologies-transactive devices-that are configured by Big Software and may be reconfigured in order to produce aesthetic images, in this case, those associated with a delightful, minimal performance of poetically implicated language that Capone has rendered as a video installation with, effectively, multi-channel presentation, and as an artifact "existing in a hybrid space between a chapbook and artists book."12

Primary Source manifested itself in the course of practice-based research, when the artist discovered, on Brown University Library's subterranean poetry shelves, a Russian language book with a striking cover design, set with a quasi-regular grid in the manner of Mondrian and De Stijl, and sparsely populated with the words of the book's title. These words, Russian in the cyrillic alphabet, were initially unreadable to Capone. Capone made use of the WordLens app on her mobile phone to try and decipher the title.

Figure 2 shows the cover and four pages from Capone's chapbook. The cover displays an image of the artist's phone, running WordLens, itself showing an acquired image of the Russian source book's cover before WordLens has attempted to provide a visualized translation into English. Transcribed and conventionally translated, this is the cover of Den' poezzii (Day of Poetry), the 1962 volume of an annual that was published by the Soviet Writer publishing house in Moscow from 1956. To the immediate right of the cover we see one of many translations offered by WordLens when it was set to translate from Russian to English. The other three images show differing configurations, and the fourth is the detail of a reading from the top right of the grid.
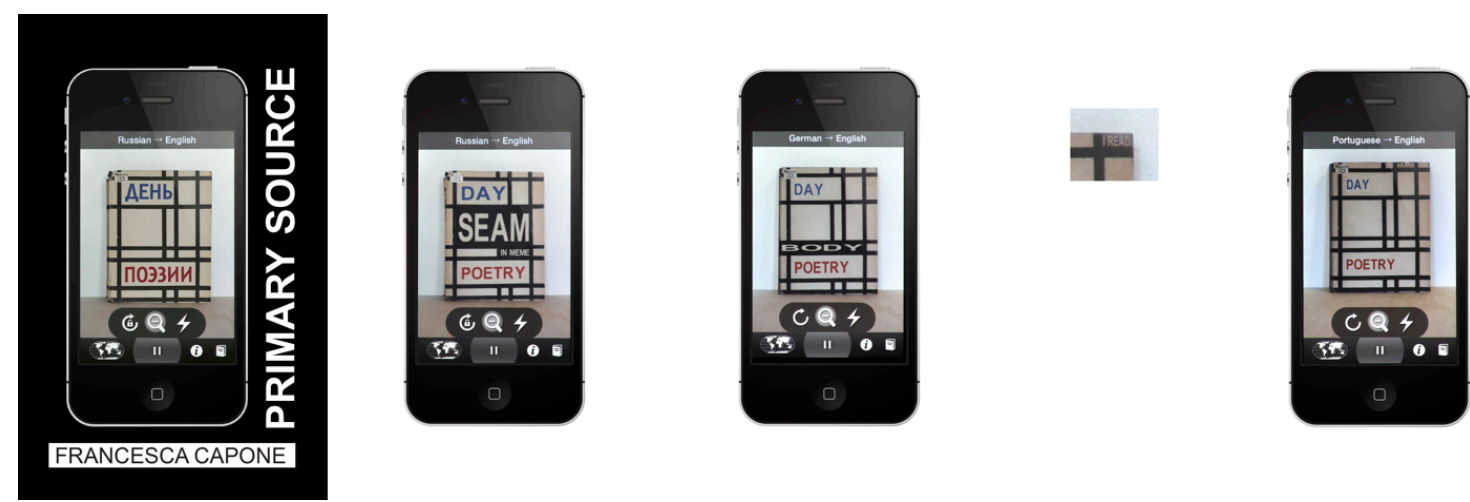

Figure 2. The cover and four pages (on two openings) from Francesca Capone's Primary Source [27].

Reproduced courtesy of the artist.

WordLens, released by Octavio Good in 2010, is an application broadly associated with so-called 'augmented reality'. Typically, such applications use the camera of a mobile device to capture images from the 'real' world and then 'augment' these images with layers of visual or textual information. WordLens tries to find and capture the images of words-the graphic forms of words in any language - and then translate these words or phrases into one of a number of possible host languages, selected by the user and would-be reader. On screen, WordLens then replaces the

12 Personal email communication, 21 March 2016 [26]. Primary Source was first publicly exhibited at the Proxy curatorial space, Providence, RI, March-April, 2015 as part of a group show entitled 'Maximum Sideline: Postscript'. Capone self-published a print-on-demand artist's book/chapbook version to coincide with the installation. On-demand print copies of this book may be ordered from the following URL: http:/ / www.lulu.com/shop/francesca-capone/primary-source/paperback/ product-22217179.html. Both video and PDF versions of the work were subsequently published online by Gaus PDF (http: /gaus-pdf.com), PDF: http:/ / www.gauss-pdf.com/post/121599676480/gpdf177gpdfe016-1-francesca-capone-primary, and video: http:/ / www.gauss-pdf.com/post/121599892473/gpdf177gpdfe016-2-francesca-capone-primary [27]. Remarks following on in the main text are adapted from an afterword that the author wrote for Capone's chapbook. 
reality-supplied word-image with the image of a supposedly corresponding-'translated'—word in the user's selected language. WordLens worked remarkably well. It was acquired by Google in 2014 and is now incorporated into Google Translate.

When WordLens was applied to the grid-embedded title of this Russian book, Capone discovered a virtual linguistic beauty in the augmented reality that it proposed to her. WordLens successfully translated the title itself, but its would-be prosthetic, word-form-seeking sensory apparatus was 'confused' by the cover's De Stijl grid. It is likely that WordLens looks for text as, itself, a more or less regular grid-like pattern, and so it also tries to 'read' what, to our non-augmented eyes, is purely formal grid, finding language-symbolic 'differences' where we do not. Moreover, the differences that WordLens sees are tiny, affected by slight movements or changes of focus and light. These cause WordLens to revise its reading continually-even when set to interpret from a single language-and, effectively, to produce an animated sequence of textual events as it reads and rereads the grid and successively augments its screen-projected reality with changing virtual text. What we see has immediate appeal for us as creatures who read. It is not simply that WordLens distorts and disturbs the visual field in a way that is merely, sensually, pleasant for us. WordLens pretends to read the image itself and there it discovers language for us in a structured field the potential symbolic understanding of which is, perhaps, expressed at a resolution or in a form that eludes our merely human visual acuity.

Capone's composite video captures the animated, flickering engagement of WordLens with translation, with actual words in a language that is unknown to her and a grid that the algorithm also reads and misreads as language. The symbolic image of her work is a synthesis of conventional translation and a number of symbolic processes that are intended to augment the human facility for translation. WordLens (re)configures translation. It is significant that it renders translation through visuality, attempting, literally, to overlay the visual forms of untranslated words that it discovers in an image with the visual forms of translated words. It configures translation as transfiguration. WordLens begins with the technology of optical character recognition (OCR) - this is one of the chief symbolic processes that it brings to Capone's reconfigured symbolic image-but then applies further natural language processing (NLP) in order to find, generate, and configure a translation of whatever it has read.

Having discovered the real-time effects of WordLens' configuration of the events or mise en scène of translation-here, Capone's desire to translate the title of a book that interested her-the artist goes on to reconfigure this mise en scène such that it reproduces the effects that she discovered-an animated engagement with the book as poetry, the cover of a particular chosen book and a poetry that underlies its poetry. This is an account of the making of the work that is now Primary Source, a symbolic image, expressed as a video installation and a hybrid print publication, and proposed as animated visual poetry, as a visualized poetics.

Read here as reconfigurationist, Capone's work also, as it happens, illustrates the precarity of contemporary artists who work with (re)configured media (mediums), who work with the increasingly predominant cultural configurations of networked computation. As we mentioned, WordLens was acquired for Google Translate in 2014. The configuration and transfiguration of translation that Capone worked with has now changed. In fact, her reconfigurationist gesture became historical before it was publicly exhibited. To understand Primary Source fully we already have to set it in a particular context - which has changed for commercially implicated reasons having little to do with aesthetic practice - rather than simply being able to appreciate it as an earlier contribution to an ongoing practice. This is too often the case with regard to work in new or digital media for the same or analogous reasons. It is, perhaps, the greatest challenge of reconfigurationist poetics—to make aesthetic artifacts that have enough cultural value and momentum so as to be able to reconfigure cultural practice itself, redirecting it away from vectors of carelessness, greed, and stupidity, toward human carefulness and careful art.

Conflicts of Interest: The author declares no conflict of interest. 


\section{References and Notes}

1. Stiegler, Bernard. What Makes Life Worth Living: On Pharmacology, English ed.Translated by Daniel Ross. Cambridge and Malden: Polity Press, 2013.

2. Stiegler, Bernard. For a New Critique of Political Economy. Cambridge: Polity, 2010.

3. Cayley, John. "Terms of Reference \& Vectoralist Transgressions: Situating Certain Literary Transactions over Networked Services." Amodern 2, 2013. Available online: http:/ / amodern.net/article/terms-of-referencevectoralist-transgressions / (accessed on 10 March 2017).

4. Cayley, John. “The Listeners.” 2015. Available online: http://programmatology.shadoof.net/?thelisteners (accessed on 20 March 2016).

5. Cayley, John. "Aurature at the End(s) of Electronic Literature." Electronic Book Review, 2017. Available online: http:/ / electronicbookreview.com/thread/electropoetics/aurature (accessed on 10 March 2017).

6. Cayley, John. "The Listeners: An instance of aurature at the end(s) of electronic literature." Dichtung-Digital, 2017, forthcoming.

7. Flusser, Vilém. Does Writing Have a Future? (Electronic mediations). Translated by Nancy Ann Roth. Minneapolis: University of Minnesota Press, 2011.

8. Flusser, Vilém. Into the Universe of Technical Images. (Electronic mediations). Translated by Nancy Ann Roth. Minneapolis: University of Minnesota Press, 2011.

9. "New Aesthetic." Available online: https:/ /en.wikipedia.org/wiki/New_Aesthetic (accessed on 20 March 2016).

10. Bridle, James. “The New Aesthetic: Waving at the Machines.” 2011. Available online: http://booktwo.org/ notebook/waving-at-machines / (accessed on 20 March 2016).

11. Cayley, John. "The Gravity of the Leaf: Phenomenologies of Literary Inscription in Media-Constituted Diegetic Worlds." In Beyond the Screen: Transformations of Literary Structures, Interfaces and Genres. Edited by Peter Gendolla and Jörgen Schäfer. Bielefeld: Transcript, 2010, pp. 199-226.

12. Valla, Clement. "Postards from Google Earth." 2010. Available online: http://www.postcards-from-googleearth.com (accessed on 20 March 2016).

13. Cloninger, Curt. "Manifesto for a theory of the 'New Aesthetic'." Mute 3 (2013): 16-27.

14. Valla, Clement. Surface Proxy. Edited by XPO Gallery. Paris: XPO Gallery, 2015.

15. Valla, Clement. "Surface Proxy." Available online: http://clementvalla.com/wp-content/uploads/2015/06/ clement_valla_surface_proxy_web.pdf (accessed on 26 March 2016).

16. Cayley, John. "Reading and Giving_-Voice and Language." Performance Research 18 (2013): 10-19. [CrossRef]

17. Derrida, Jacques. Of Grammatology, Corrected ed. Translated by Gayatri Chakravorty Spivak. Baltimore and London: Johns Hopkins University Press, 1997.

18. Sondheim, Alan. "Introduction: Codework." American Book Review 22 (2001): 1-4.

19. Cayley, John. "The Code Is Not the Text (Unless It Is the Text)." Electronic Book Review, 2002. Available online: http:/ / www.electronicbookreview.com/thread/electropoetics/literal (accessed on 10 March 2017).

20. Hicks, William P. “Esopo.” Personal email communication, 29 March 2016.

21. Montfort, Nick. \#! [Shebang]. Denver: Counterpath, 2014.

22. Cayley, John. "Poetry and Stuff: A Review of \#!" Electronic Book Review, 2015. Available online: http://www.electronicbookreview.com/thread/electropoetics/shebang (accessed on 23 September 2015).

23. Montfort, Nick. "ppg256 Series: Perl Poetry Generators in 256 Characters." 2008. Available online: http:/ /nickm.com/poems/ppg256.html (accessed on 10 March 2017).

24. Cayley, John. Image Generation. London: Veer Books, 2015.

25. Cayley, John, and Daniel C. Howe. "The Readers Project." 2009. Available online: http:/ /thereadersproject. org (accessed on 25 March 2016).

26. Personal email communication, 21 March 2016.

27. Capone, Francesca. Primary Source. Providence: Self-Published Artist's Book, 2015. And then online by Gauss PDF (see note).

(C) 2017 by the author. Licensee MDPI, Basel, Switzerland. This article is an open access article distributed under the terms and conditions of the Creative Commons Attribution (CC BY) license (http:/ / creativecommons.org/licenses/by/4.0/). 